\title{
NEW RESULTS AND OLD PROBLEMS IN FINITE TRANSFORMATION GROUPS ${ }^{1}$
}

\author{
P. A. SMITH
}

We shall be concerned with the topology of finite transformation groups on spaces of relatively simple character. This represents a rather small corner in the general theory, but the problems which one finds here seem to be of some interest and difficulty. By disposing of various low-dimensional cases, we shall try to show where the real difficulties begin.

1. Definitions. A transformation group $(G, X)$ consists of a group $G$ acting on a topological space $X$ to form a group of homeomorphisms of $X$ onto itself. It will be understood throughout this paper that $G$ is finite. For a given transformation group or "action" $(G, X)$ and subset $H \subset G$, we denote by $F(H ; G, X)$ the fixed-point set of $H$-that is, the points $x$ such that $h x=x$ for $h \in H$. We may of course denote this set simply by $F(H)$ when only one action is being considered. An action $(G, X)$ is $g$-free if $F(g)=\varnothing$, free if it is $g$-free when $g \neq 1$, and semi-free if $F(G)=\varnothing$. Let $X^{r}$ be the union of the fixed-point sets $F(g), g \neq 1$, and let $X^{f}=X-X^{r}$. Since $g F(h)=F\left(g h g^{-1}\right)$, the closed set $X^{r}$ is invariant under the transformations $x \rightarrow g x$. Hence $G$ acts on $X^{r}$ (if $X^{r}$ is not empty) hence also on $X^{f}$. The action $\left(G, X^{f}\right)$ is free but the action $\left(G, X^{r}\right)$ is not free. We call $X^{f}$ the free part of $X$ and $X^{r}$ the restricted part; $\left(G, X^{r}\right)$ may be called the restricted part of the action $(G, X)$. An action $(G, X)$ is effective if $F(g) \neq X$ when $g \neq 1$. In a given action, the set $N$ of elements $g$ with $F(g)=X$ is a normal subgroup of $G$ and there is induced an action $(G / N, X)$ which is effective.

The sets $G x, x \in X$, are the orbits of $(G, X)$. They form a decomposition of $X$ and the corresponding decomposition space, called the orbit space of the action, is denoted by $X / G$. The stability group $G_{x}$ of $x$ consists of all $g$ such that $g x=x$.

An action $(G, X)$ is of class $C^{k}$ if $X$ is a manifold of class $C^{k}$ and the functions $x \rightarrow g x$ are of class $C^{k}$. When $k=0$ we shall drop the manifold condition on $X$; every action is then of class $C^{0}$. A differentiable action is a $C^{1}$-action. $(G, X)$ is orthogonal if $X$ is a euclidean sphere or an open submanifold of a euclidean space and the trans-

An address delivered before the Summer Meeting of the Society in Salt Lake City on September 3, 1959 by invitation of the Committee to Select Hour Speakers for Annual and Summer Meetings; received by the editors April 6, 1960.

1 This work has been supported by the Office of Naval Research. 
formations $x \rightarrow g x$ are orthogonal. An action $(G, X)$ which is of class $C^{k}$ (or orthogonal) is triangulated if $X$ carries a triangulation which is of class $C^{k}$ (or orthogonal) and is compatible with the action. An orthogonal triangulation here means one in which the cells are simplexes if $X$ is a euclidean space, and geodesic simplexes if $X$ is a euclidean sphere.

2. Isomorphism classes. An isomorphism $\phi:(G, X) \rightarrow\left(G^{\prime}, X^{\prime}\right)$ of two actions, both of class $C^{k}$ or orthogonal, consists of an isomorphism $G \rightarrow G^{\prime}$ and a homeomorphism $X \rightarrow X^{\prime}$, of class $C^{k}$ or orthogonal (both mappings being bijective) such that $g x \rightarrow g^{\prime} x^{\prime}$ whenever $g \rightarrow g^{\prime}, x \rightarrow x^{\prime}$. For a given $G$ and $X$, the actions $(G, X)$ of class $C^{k}$ fall into isomorphism classes the totality of which we denote by $I^{k}(G, X)$. Let $I^{\text {or }}(G, X)$ be the corresponding sets for orthogonal actions. Since every action and isomorphism of class $C^{k}$ can be regarded as of class $C^{0}$, we have a natural mapping $I^{k}(G, X) \rightarrow I^{0}(G, X)$. Similarly we have $I^{\text {or }}(G, X) \rightarrow I^{k}(G, X)$.

For each $G$, the mappings

$$
I^{\mathrm{or}}\left(G, S_{n}\right) \rightarrow I^{k}\left(G, S_{n}\right) \rightarrow I^{0}\left(G, S_{n}\right)
$$

are bijective when $n=0,1,2$. This is trivial for $n=0$ and easy for $n=1$; the proof for $n=2$ is due to Kérékjártó [12].

The mappings $I^{\text {or }}\left(Z_{2}, S_{n}\right) \rightarrow I^{0}\left(Z_{2}, S_{n}\right)$ are not all surjective when $n \geqq 3$. Bing [1], for example, has constructed an action $\left(Z_{2}, S_{n}\right)$ in which $F\left(Z_{2}\right)$ is a topological 2-sphere, and the free part of $S_{3}$ is not the disjoint union of two 3-cells as it would have to be were the action isomorphic to an orthogonal one. Actions $\left(Z_{2}, S_{n}\right)$ not isomorphic to orthogonal ones and with arbitrarily large $n$, have been given by J. H. C. Whitehead [25]. On the other hand, Hirsch and Smale [10] have shown that every action $\left(Z_{2}, S_{3}\right)$ which admits exactly two fixed points ${ }^{2}$ is isomorphic to an orthogonal one and G. R. Livesay (not yet published) showed that the same is true of every free $\left(Z_{2}, S_{3}\right)$. An example of Floyd [7] showed that $I^{\text {or }}\left(Z_{6}, S_{41}\right) \rightarrow\left(I^{0}\left(Z_{6}, S_{41}\right)\right.$ is not surjective, in fact the image of $I^{\text {or }}$ does not even contain all members of $I^{0}$ which have triangulable representatives. In the triangulated action $\left(Z_{6}, S_{41}\right)$ constructed by Floyd, $F\left(Z_{6}\right)$ is not homeomorphic to a euclidean sphere.

Call two orthogonal actions $\left(G, S_{n}\right)$ combinatorially isomorphic if they admit triangulations which are carried one into the other by

${ }^{2}$ Other supports can be used. Concerning the definitions and statements in this paragraph see $[18]$ or $[1 ; 19 ; 20]$. 
some (not necessarily orthogonal) isomorphism. It is easy to see that orthogonal isomorphism implies combinatorial isomorphism and hence we have a natural mapping $I^{\text {or }}\left(G, S_{n}\right) \rightarrow I_{c}^{\text {or }}\left(G, S_{n}\right)$ where $I_{c}^{\text {or }}$ consists of the combinatorial isomorphism classes. These mappings are injective when $G=Z_{m}, m>1$. This was proved by de Rham [16] for free actions and the general case follows readily.

Not much more is known at present about the natural mappings of isomorphism classes.

3. Joins. Two transformation groups $(G, X),\left(G^{\prime}, X^{\prime}\right)$ determine in a natural way an action $\left(G \times G^{\prime}, X \circ X^{\prime}\right)$ on the join $X \circ X^{\prime}$. If $G=G^{\prime}$ we may restrict the action of $G \times G$ to the diagonal of $G \times G$ and obtain an action $\left(G, X \circ X^{\prime}\right)$. We shall denote this action by $(G, X) \circ\left(G, X^{\prime}\right)$.

The join $S_{m} \circ S_{n}$ of two euclidean spheres is homeomorphic to $S_{m+n+1}$. In fact one can assign the structure of a euclidean $(m+n+1)$ sphere to $S_{m} \circ S_{n}$ in such a way that $\left(G, S_{n}\right) \circ\left(G, S_{m}\right)$ is orthogonal and uniquely determined up to (orthogonal) isomorphism. The multiplication defined by $\left(G, S_{n}\right) \circ\left(G, S_{m}\right)$ is associative up to isomorphism.

Suppose that $G$ is abelian and $\left(G, S_{n}\right)$ orthogonal. Then from elementary properties of real representations there is a "decomposition"

$$
\left(G, S_{n}\right)=(G, S) \circ\left(G, S^{\prime}\right) \circ \cdots \circ\left(G, S^{(k)}\right)
$$

where $S, S^{\prime}, \cdots$ are spheres and where the factors are orthogonal and can not themselves be factored; this decomposition is unique up to isomorphism and the order of the factors. The transformation groups and isomorphism in (1) can of course be regarded as being of class $C^{0}$, but from the $C^{0}$ point of view it is not known whether uniqueness holds. The question of $C^{0}$-uniqueness for the decomposition of free orthogonal actions $\left(Z_{m}, S_{2 n+1}\right), m>2$, is essentially equivalent to the problem of classifying lense spaces, which are the orbit spaces of such actions. De Rham [16] showed that uniqueness does hold for such actions if "isomorphism" is taken to mean "combinatorial isomorphism." This implies a combinatorial classification of lense spaces.

The examples of Bing, Floyd and Whitehead in $\$ 2$ show that not every abelian $\left(G, S_{n}\right)$ (of class $C^{0}$ ) has a decomposition (1) even if it is triangulable. It is in fact doubtful that a decomposition necessarily exists in the differentiable case. For one reason, the sets $F(g)$ for the right member of (1) with differentiable factors, are homeomorphic 
to spheres whereas it is doubtful if this is true of the set $F(g)$ for every differentiable action $\left(G, S_{n}\right)$ although so far as the writer knows, no counter-example has been constructed.

4. Effective actions in spheres. Let $E^{k}(X)$ denote the totality of finite groups $G$ such that there exist effective actions $(G, X)$ of class $C^{k}$, and let $E^{\text {or }}(X)$ denote the corresponding set for orthogonal actions. It is known that $E^{0}\left(S_{n}\right)=E^{\text {or }}\left(S_{n}\right)$ when $n=0,1,2$ but it is not known whether this is true when $n>2$. (We shall consider the case $n=2$ in $\$ 7$.) It should be remarked that while the members of $E^{\text {or }}\left(S_{n}\right)$ can be listed when $n \leqq 3$ (Seifert and Threlfall [17]), this is not the case when $n>3$. We note also that every group $G$ can act effectively on some $S$ which has the same homotopy type as $S_{n}$. In fact let $S=S_{n} \cup(p \circ G)$ where $p$ is a point of $S_{n}$ and let $(G, S)$ be defined by $g x=x, x \in S_{n}, g(p \circ h)=p \circ g h$. This action is effective, and $S$ is retractible to $S_{n}$ by deformation.

No example is known of an effective action $\left(G, S_{n}\right)$ where $G$ can not act effectively and orthogonally on $S_{n}$, and one might conjecture that no such action exists. At any rate, one can show that none exists in which $G$ is an abelian p-group. This is a straightforward consequence of the next proposition.

By a homology $n$-sphere over $A$ we shall mean a locally compact finite dimensional Hausdorff space $X$ such that $H^{*}(X, A)=H^{*}\left(S_{n} ; A\right)$ where $H^{*}$ means cohomology with compact supports. ${ }^{2}$ It is to be understood that $S_{0}$ consists of two points and that the empty set is a homology $(-1)$-sphere. If $X$ is a homology $n$-sphere over $Z_{p}, p$ a prime, and if $Z_{p}$ acts on $X$, then $F\left(Z_{p}\right)$ is a homology $m$-sphere over $Z_{p}$ and $-1 \leqq m \leqq n ; n-m$ is even if $p>2$. The example above shows that $m$ can equal $n$ even if the action is effective, but this possibility can be ruled out by imposing local conditions. Call $X$ a generalized $n$-sphere over a principal ideal domain $A$ if it is a homology $n$-sphere over $A$ and a generalized $n$-manifold over $A$. A generalized 0 -sphere over $A$ consists of two points. If $X$ is a generalized $n$-sphere over $Z_{p}$, then for any action $\left(Z_{p}, X\right), F\left(Z_{p}\right)$ is a generalized $m$-sphere over $Z_{p}$ and if the action is effective, $m<n$. If $X$ is a homology $n$-sphere over $Z_{2}$ and $H^{n}(X ; Z)$ is finitely generated, one can distinguish between actions $\left(Z_{2}, X\right)$ which preserve orientation and those which reverse orientation. If orientation is preserved then $n-m$ is even.

Let $X$ be a generalized $n$-sphere over $Z_{p}, p$ a prime, and let $G=Z_{p} \times \cdots \times Z_{p}, s$ factors, act effectively on $X$. If $p>2$, then $s \leqq(n+1) / 2$. In any case $s \leqq n+1$.

Suppose $p>2$. If $s=1$, there is nothing to prove. Assume $s \geqq 2$. We shall show that there exist subgroups $G=G^{1} \supset G^{2} \supset \cdots \supset G^{s-1}$, each 
of index 1 in the preceding, and generalized spheres $X=X^{n}$, $X^{n-1}, \cdots, X^{s-1} \neq \varnothing$ of strictly decreasing dimension such that $G^{i}$ acts on $X^{i}$ (i.e. $g X^{i}=X^{i}$ whenever $g \in G^{i}$ ) and does so effectively. Suppose in fact that for some $i$ with $i<s-1, G^{1}, \cdots, G^{i}$ and $X^{1}, \cdots, X^{i}$ have been defined. The relation $i<s-1$ implies that $G^{i}$ is noncyclic and therefore $\left(G^{i}, X^{i}\right)$ can not be free [22]. Hence there exist cyclic subgroups $H$ of $G^{i}$ such that $F(H)\left(=F\left(H ; G^{i}, X^{i}\right)\right)$ is nonempty. Among the cyclic subgroups $H$ of $G^{i}$ for which $F(H)$ $\neq \varnothing$, let $H^{1}$ be one such that $F\left(H^{1}\right)$ is maximal i.e. is not properly contained in some $F(H)$. Take $G^{i+1}$ to be a nontrivial cyclic subgroup of $G^{i}$ such that $G^{i+1} \cap H^{1}=\{1\}$, and take $X^{i+1}$ to be $F\left(H^{1}\right)$ noting that $X^{i+1} \neq \varnothing$. Since $G$ is abelian, $G^{i+1}$ acts on $X^{i+1}$ and it only remains to be proved that $\left(G^{i+1}, X^{i+1}\right)$ is effective. If not, there is a nontrivial cyclic subgroup $H^{\prime}$ of $G^{i+1}$ leaving $X^{i+1}$ pointwise fixed. This means that the fixed-point set of $H^{\prime}$, acting on $X^{i}$, contains $X^{i+1}$, i.e. that $F\left(H^{\prime}\right) \supset F\left(H^{1}\right)$. Hence by maximality, $F\left(H^{\prime}\right)=F\left(H^{1}\right)$ and therefore the nontrivial elements of $H^{\prime} H^{1}$ all have the same fixed-point set, namely $F\left(H^{1}\right)$ and therefore [22] $H^{\prime} H^{1}$ is cyclic, hence $H^{\prime}=H^{1}$. But this is impossible since $H^{\prime} \subset G^{i+1}$. Now the $X$ 's are generalized spheres over $Z_{p}$, and each is the fixed-point set of an action on the preceding by a cyclic subgroup of $G$. The "dimensions" of the $X$ 's form a strictly decreasing sequence ending with that of $X^{s-1}$, call it $k$. Then $0 \leqq k \leqq n-(s-1)$, hence $x \leqq n+1$. If $p>2$, the dimensions decrease by even jumps, hence $0 \leqq k \leqq n-2(s-1)$, $s \leqq(n+1) / 2$.

5. Groups of order $p q$. Let $G(p, q, k)$ denote the nonabelian group of order $p q$ defined by $s^{q}=t^{p}=1, s t s^{-1}=t^{k}$, with $p, q$ distinct primes, $p>2, k \neq 1, k^{q}=1 \bmod p$.

Call an action $(G, X)$ regular if the sets $F(g), g \in G$, are manifolds. If $(G, X)$ is regular and $X$ is orientable, all the manifolds $F(g)$ are orientable [20]. Consider an action $\left(G_{p q}, S_{n}\right)$ where $G_{p}=G(p, q, k)$. Let $S_{n}$ and $F(t)$ be oriented. $G_{p q}$ acts on $F(t)$ since the subgroup generated by $t$ is normal. Call the action $\left(G_{p q}, S_{n}\right)$ concordant if $s$ preserves or reverses both orientations, agreeing that the orientation of $F$ is preserved if $F=\varnothing$. The action is necessarily concordant if $q>2$. Let $r=\operatorname{dim} F(t)$ agreeing that $\operatorname{dim} \varnothing=-1$. Since $p>2, n-r$ is even. A regular $s$-free action in which $q=2$ is necessarily concordant.

Suppose $G(p, q, k)$ acts regularly, effectively and concordantly on $S_{n}$. Then $n=\mathrm{r} \bmod 2 q$. The hypothesis of regularity can be omitted if the action is $t$-free.

Proof. Let $F=F(t)$, and let $S_{n}$ and $F$ be oriented. To each trans- 
formation $T: S_{n} \rightarrow S_{n}$ of period $p$ having $F$ as fixed-point set, there is associated [11], Appendix B an element of $Z_{p}$, denoted by ind $T$, $\operatorname{such}^{3}$ that (1) ind $T^{k}=k^{(r-n) / 2}$ ind $T$ where $r=\operatorname{dim} F$; (2) if $S$ is a homeomorphism $\left(S_{n}, F\right) \rightarrow\left(S_{n}, F\right)$ which preserves or reverses both orientations, then ind $\left(S T S^{-1}\right)=$ ind $T$. Let ind $t=\operatorname{ind} T$ where $T$ is the transformation $x \rightarrow t x$. Then

$$
\text { ind } t=\text { ind } s t s^{-1}=\text { ind } t^{k}=k^{(r-n) / 2}
$$

so that $k^{(r-n) / 2}=1 \bmod p$. Since $k^{q}=1 \bmod p$ and $k \neq 1$, we see that $q$ must divide $(r-n) / 2$, hence $r-n=0 \bmod 2 q$.

The preceding proposition restricts the cases in which effective action can occur. We mention the following instances:

(a) If $q>2, G(p, q, k)$ can act $t$-freely on $S_{n}$ only if $n+1=0 \bmod 2 q$.

(b) If $q>2, G(p, q, k)$ can not act effectively and regularly on $S_{2}, S_{3}, S_{4}$. If there exists such an action on $S_{5}$ it must be $t$-free.

(c) If $q>2, G(p, q, k)$ can not act differentiably and effectively on $S_{6}$. For, suppose there is such an action. Since $6-r \geqq 2 q \geqq 6$, and $6-r$ is even, we have $r=0$. Hence $F(t)$ consists of two points. Since $q$ is odd, both points are fixed under $s$. Hence $F\left(G_{p q}\right) \neq \varnothing .\left(G_{p q}, S_{6}\right)$ induces an effective orthogonal action on the tangent vector space of any point of $F\left(G_{p q}\right)$ hence an effective regular action of $\left(G_{p q}, S_{\mathfrak{5}}\right)$, which is impossible by (b).

If $q=2$, then $k=-1$ and $G(p, 2,-1)$ is the dihedral group $G_{2 p}$ of order $2 p$. Milnor [13] showed that $G_{2 p}$ can not act freely on any $S_{n}$. Of course $G_{2 p}$ can not act freely and orthogonally on $S_{n}$ for the reason that since $s$ is of order 2 and $F(s)$ is empty, $s$ would be represented by the matrix $-I$ and would therefore permute with $t$.

In the cases which have not been excluded by the preceding remarks, it is not known whether $G(p, q, k)$ can act effectively on a given sphere. As Milnor remarked, the simplest unsolved case for free actions is the following. Can $G(7,3,2)$ of order 21 act freely on $S_{5}$ ? Zassenhaus [28] showed that $G(p, q, k)$ can not act freely and orthogonally on any $S_{n}$.

In the case of actions which are not free, perhaps the simplest unsolved case is the following. Suppose $\left(G_{2 p}, S_{4}\right)$ is regular and concordant. Then $4=r \bmod 4$, hence $r=0$. Can this actually occur? Specifically, can the dihedral group of order 6 (i.e. $G(3,2,-1)$ ) act on $S_{4}$ in such a way that $F(s)=\varnothing$ and $F(t)$ consists of two points? No such orthogonal action is possible. ${ }^{4}$

${ }^{3}(1)$ is not explicitly stated in [11] but can easily be verified.

${ }^{4}$ A related question: let $\left(G, S_{n}\right)$ be a differentiable action such that $F(G)$ consists of two points $x, y$. Are the induced actions $\left(G, T_{x}\right),\left(G, T_{y}\right)$ on the tangent vector spaces isomorphic? 
Most of the results in this section remain true if $S_{n}$ is an $n$-manifold which is an integral homology $n$-sphere. It remains true for example that the dihedral group can not act freely on $S_{n}$. On the other hand, recent results of Swan [24] show that such actions do exist on homology spheres which are not manifolds.

6. Groups with periodic cohomology. It is well known [3, p. 358] that if $G$ acts freely on a sphere of odd dimension $n$, then $G$ has cohomology of period $n+1$. This means that $\left[3\right.$, p. 260] $\hat{H}^{n+1+k}(G ; A)$ $=\hat{H}^{k}(G, A), k=0,1, \cdots$ for every coefficient module $A$. The only properties of the $\hat{H}^{k}$ which we shall need are the following $[3, \mathrm{p}$. 237 , p. 250]:

$$
\begin{aligned}
\hat{H}^{0}(G, Z) & =Z_{d}, \quad d=[G: 1] \\
\hat{H}^{2}(G, Z) & =\operatorname{Hom}\left(G /[G, G], Z_{d}\right)
\end{aligned}
$$

It is assumed in these formulas that $G$ acts trivially on $Z$.

REMARK. If $\hat{H}^{0}(G, Z)=\hat{H}^{2}(G, Z)$ with trivial action on $Z$, then $G=Z_{d}$. For, $\operatorname{Hom}\left(G /[G, G], Z_{d}\right)=Z_{d}$ implies easily that $G /[G, G]$ contains an element of order $d$ and this in turn implies that $G=Z_{d}$ since $G$ and $Z_{d}$ are both of order $d$.

We shall need the following mild generalization of the theorem of periodic cohomology.

Let $X$ be an integral homology $n$-sphere and $Y \subset X$ an integral homology $m$-sphere with $m \leqq n-2$. If $G$ acts on $X$ in such a way that $g Y=Y$ for $g \in G$ and $G$ acts freely on $X^{\prime}=X-Y$, then $G$ has cohomology of period $n-m$.

PROOF. There exists a spectral sequence $E_{r}[3$, p. 354] or [18, Chapter IV] such that $E_{\infty}$ is associated with the cohomology of the orbit space $X^{\prime} / G$ over a coefficient module $A$ and $E_{2}^{p, g}$ $=H^{p}\left(G, H^{q}\left(X^{\prime}, A\right)\right)$. Cohomology here is taken with compact supports and is reduced in the dimension 0 . From the Künneth relations, $H^{n}(X, A)=A, H^{h}(X, A)=0, h \neq n$. Hence from the cohomology sequence for $(X, Y)$ we have $H^{*}\left(X^{\prime}, A\right)=H^{n}\left(X^{\prime}, A\right) \oplus H^{m+1}\left(X^{\prime}, A\right)$ $=A \oplus A$. Thus $E_{2}^{p, q}$ is nontrivial only when $q=n, m+1$. Using $E_{r+1}$ $=H\left(E_{r}\right)$ and the fact that $d_{r}$ is of bi-degree $(r, 1-r)$, we find that $E_{2}^{s n}=\cdots=E_{r}^{s n}$ and $E_{2}^{s, m+1}=\cdots=E_{r}^{s, m+1}$ where $r=n-m, s=0$, $1, \cdots$. We have

$$
d_{r}^{s, n}: E_{r}^{s, n} \rightarrow E_{r}^{s+n-m, m+1} \quad(s=0,1, \cdots) .
$$

We assert that $d_{r}^{s, n}$ is bijective when $s \geqq 1$. For, $E_{2}^{p, m+1}, p \geqq n-m$, evidently consists of permanent cocycles of total degree $\geqq n+1$. But $E_{\infty}$ gives the cohomology of $X^{\prime} / G$ which can be shown to be trivial 
in dimensions exceeding $n$ (in order not to stop for this point we can assume that $\operatorname{dim} X=n$ in which case it is trivial). Let $x \in E_{r}^{s+n-m, m+1}$. By what has just been said, the image of $x$ in $E_{\infty}$ is zero. Assume $x \neq 0$. Then $x$ must be "killed off" in passage to $\infty$ which means that $x$ has an image in some $E_{t}, t \geqq r$, of the form $d_{t} y, y \neq 0$. Using the fact that $d_{t}$ is of bidegree $(t, 1-t)$ we see that this can happen only if $t=r$ and $y \in E_{r}^{s, n}$. It follows that (1) is surjective. Suppose $s>1$. If (1) is not injective $E_{r}^{s, n}$ would contain a nonzero permanent cocycle, which, being of total degree $\geqq n+1$, must be killed off by an element in some $E_{s}^{u, v}$ where $v>n$ whereas, all such elements being images of elements in $E_{2}^{u, v}$ are zero, which proves the assertion. It follows from this and the fact that $H^{s}=\hat{H}^{s}$ when $s \geqq 1$, that $\hat{H}^{s}(G, A)$ $=\hat{H}^{n-m+s}(G, A)$ for every $A$ when $s \geqq 1$. It must therefore hold also for $s=0[3$, p. 358].

Using the remark in the second paragraph of this section we have the following

Corollary. If $X$ is an integral homology n-sphere and $(G, X)$ an action such that $X^{f}=X-F(G)$ and if $F(G)$ is an integral homology $(n-2)$-sphere, then $G$ is cyclic.

7. Actions on a generalized 2-sphere. Let $\Omega$ be the set of orbits of an action $(G, X)$ where $X$ is finite (discrete). Since $G_{g x}=g G_{x} g^{-1}(\S 2)$ we see that $\left[G_{x}: 1\right]$ is constant on each orbit $\omega$. Let $\nu(\omega)=\left[G_{x}: 1\right]_{x \in \omega}$. Let $N=[G: 1]$ and let $\phi(g)$ be the number of points in $F(g)$. Then

$$
\sum_{g \neq 1} \phi(g)=N \sum_{\omega \in \Omega}(1-1 / \nu(\omega)) .
$$

This is shown by counting the number $P$ of pairs $(g, x)$ such that $g \neq 1, g x=x$. There are $\phi(g) x$ 's paired with each $g \neq 1$, hence the left members of (1) equals $P$. For $x$ in a given orbit $\omega$, there are $\nu(\omega)-1$ $g$ 's paired with $x$; hence to $\omega$ there correspond $(\nu(\omega)-1) n(\omega)$ pairs where $n(\omega)$ is the number of elements in $\omega$. Now $n(\omega)$ equals the number of cosets of $G_{x}, x \in \omega$, hence $n(\omega) \nu(\omega)=N$. Hence the number of pairs corresponding to $\omega$ is $N(\nu(\omega)-1) / \nu(\omega)$ and therefore $P$ equals the right member of (1).

Let $G$ act on a finite set $X$ such that $F(g)$ consists of a single point when $g \neq 1$ and each $G_{x}$ is nontrivial. Then $X$ consists of just one point.

For, with $\phi(g)=1, g \neq 1$, (1) becomes

$$
1-1 / N=\sum(1-1 / \nu(\omega)) .
$$

$G_{x} \neq\{1\}$ implies $\nu(\omega) \geqq 2$ for every $\omega$. Hence the right member of (2) is $>1$ if there are two or more terms in the sum whereas the left member is smaller than 1 . We conclude that there is just one orbit $\omega$ 
and that $\nu(\omega)=N$. Hence $G_{x}=G$ for each $x$ so that $F(G)=X$. Therefore $F(g)=X$ for every $G$, hence $X$ consists of one point.

Consider now the subset $E^{\mathrm{rot}}\left(S_{2}\right)$ of $E^{\mathrm{or}}\left(S_{2}\right)$ consisting of those groups which can act effectively as rotation groups on $S_{2}$.

If there exists a $(G, X)$ with finite $X$ such that every $G_{x}$ is cyclic and nontrivial and every $F(g), g \neq 1$, consists of two points, then $G \in E^{\mathrm{rot}}\left(S_{2}\right)$.

In fact, a classical argument [27, p. 17] based on (2) shows that if $(G, X)$ has the stated properties then $(G, X)$ can be identified with the restricted part ( $\$ 1$ ) of an effective action of $G$ in $S_{2}$, in which the transformations $x \rightarrow g x$ are rotations.

Let $X$ be an integral homology 2-sphere. If the restricted set $X^{r}$ of an action $(G, X)$ is finite and each set $F(g), g \in G$, is nonempty, then $G$ is a member of $E^{\text {rot }}\left(S_{2}\right)$.

It is sufficient to show that the restricted part $\left(G, X^{r}\right)$ satisfies the hypothesis of the preceding proposition. Let $g$ be an element of $G$ of prime order $p$. Then $F(g)$ is a homology 0 -sphere over $Z_{p}$. But as a subset of $X^{r}, F(g)$ is finite and therefore consists of exactly two points. It follows readily that every $F(g), g \neq 1$, consists of two points. Since each point in $X^{r}$ is fixed under some $g \neq 1, G_{x}$ is nontrivial when $x \in X^{r}$. It remains to be shown that $G_{x}, x \in X^{r}$, is cyclic. Let $x$ be a point in $X^{r}$, and consider the action $\left(G_{x}, X\right)$. Let $X_{x}^{r}$ be the restricted part of $X$ in this action. Evidently $x \in X_{x}^{r}$ and $G_{x}$ acts on the set $X^{\prime}=X_{x}^{r}-\{x\}$ which is nonempty. Each element of $G_{x}$ different from 1 leaves just two points of $X$ fixed, one of which is $x$, hence leaves one point of $X^{\prime}$ fixed. Moreover, if $x^{\prime} \in X^{\prime}$ then at least one $g$ in $G_{x}$ different from 1 leaves $x^{\prime}$ fixed. Therefore by the first proposition in this section $X^{\prime}$ consists of a single point and so in the action $\left(G_{x}, X\right)$, $F\left(G_{x}\right)$ consists of two points and is therefore an integral homology 0 -sphere. Moreover, the free part of $X$ in this action is $X-F\left(G_{x}\right)$. Hence by the corollary in $\S 6, G_{x}$ is cyclic.

COROLlaRy. If $G$ acts effectively on an integral generalized 2-sphere so that the transformations $x \rightarrow g x$ preserve orientation, then $G$ is a member of $E^{\text {rot }}\left(S_{2}\right)$.

8. Cyclic actions on 3-spheres. Let $L$ be the restricted part of $S_{3}$ for an action $\left(Z_{m}, S_{3}\right)$ and assume that $L$ is the disjoint union of $k$ simple closed curves, $k \geqq 1$. If the action is orthogonal, $k$ is either 1 or 2. This remains true if orthogonality is replaced by the hypothesis that each component $J$ of $L$ is unknotted in the sense that $\pi_{1}\left(S_{3}-J\right)$ $=Z$. Whether or not a component of $L$ can actually be knotted is an open question; Montgomery and Samelson [14] have shown that certain types of knots are not possible. 
Let $m=p q r$ where $p, q, r$ are distinct odd primes and let $J_{p}=F\left(Z_{p}\right)$, etc. If $J_{p}, J_{q}, J_{r}$ are disjoint simple closed curves, each is knotted.

Proof. $Z_{m}$ acts on each $J$. Suppose that $J_{p}$ is unknotted: $\pi_{1}(U)=Z$, $U=S-J_{p}, S=S_{3}$. The universal covering $\tilde{U}$ of $U$ is a 3-manifold which is aspherical since $U$ is aspherical [15], hence is acyclic with respect to integral homology. Hence the fixed-point set of any transformation $\widetilde{U} \rightarrow \widetilde{U}$ of odd prime period is a nonempty manifold of dimension $r$ where $0 \leqq r<3$ and $3-r$ is even; hence $r=1$. The fixedpoint set will moreover be connected and noncompact and is therefore a line, i.e. a topological image of $E_{1}$. Let $\phi$ be the projection $\tilde{U} \rightarrow U$ and let $\tilde{J}_{q}=\phi^{-1} J_{q}, \tilde{J}_{r}=\phi^{-1} J_{r}$. Let $x \in J_{q}$. We can think of $\tilde{U}$ as consisting of the equivalence classes of paths in $U$ based at $x$. There is an obvious action of $Z_{q}$ on $\tilde{U}$ defined by the action of $Z_{q}$ on these paths. Since $J_{q}, J_{r}$ are invariant under $Z_{p}$, so are $\tilde{J}_{q}, \tilde{J}_{r}\left(\tilde{J}_{r}\right.$ for example consists of the equivalence classes of paths based at $x$ and ending at points of $J_{r}$, and is therefore invariant). Now each component $\tilde{K}$ of $\tilde{J}_{q}$ is a line or a simple closed curve. But $\tilde{K}$ can not be a s.c.c. because $\phi K$, which equals $J_{q}$, would be null-homotopic in $U$, hence in the action of $Z_{q}$ on $U, J_{q}$ would be an invariant s.c.c. which bounds in $U$, and no such curve exists. ${ }^{5}$ Now $Z_{p}$ acts on the equivalence classes of loops in $U$ based at $x$, namely on $\pi_{1}(U)=Z$ and since $q$ is odd, this action is trivial. These loops can be thought of as giving the points $\tilde{x}$ which cover $x$. Hence $Z_{q}$ leaves each cover $\tilde{x}$ of $x$ fixed. Each component $\tilde{K}$ of $\tilde{J}_{q}$ contains an $\tilde{x}$ and is therefore invariant un$\operatorname{der} Z_{q}$. Thus $Z_{q}$ acts on $\tilde{K}$ and since $q \neq 2$ and $\tilde{K}$ is a line, the action is trivial. We conclude that each point of $\tilde{J}_{q}$ is fixed under $Z_{q}$. Conversely, a point of $\tilde{U}$ fixed under $Z_{q}$ must cover a point of $J_{q}$, hence is in $\widetilde{J}_{q}$. Thus $\widetilde{J}_{q}$ is the fixed-point set of $Z_{q}$ acting on $\tilde{U}$, hence is a line. In the same way, $\tilde{J}_{r}$ is a line. But $Z_{q}$ acts on $\tilde{J}_{r}$ and $q$ being odd, the action is trivial. Hence $\tilde{J}_{r}$ consists of fixed points of $Z_{q}$ hence $\widetilde{J}_{r} \subset \tilde{J}_{q}$ which implies $J_{r} \subset J_{q}$, a contradiction.

9. Acyclic spaces. No nontrivial finite group can act freely on a euclidean space or on a closed euclidean ball. The situation with regard to semi-free actions on such spaces stands about as follows. (a) Greever [9] showed that no group of order less than 60 and different from 36 and no abelian group of order less than 210 can act semifreely on a closed ball; (b) Floyd and Richardson [8] showed that there exists a triangulable semi-free action of $A_{5}$ on a closed ball of

5 The existence of such a curve together with the fact that $H_{n}\left(U, Z_{p}\right)=0$ for $n \geqq 2$ would imply the existence of a fixed point for the action $\left(Z_{p}, U\right)$ by essentially the same argument as used in the proof of Theorem $\mathrm{I}(\alpha)$ in [21]. 
suitably high dimension, where $A_{5}$ is the group of even permutations on 5 letters, hence of order 60 ; (c) for $m \geqq 2, Z_{m}$ can not act semifreely on $E_{n}$ if $n \leqq 4$; (d) there exists no differentiable semi-free action $Z_{p q}$ on $E_{n}$ if $p, q$ are primes and $n \leqq 6$; (e) there exists an orthogonal semi-free action of $Z_{p q}$ on a contractible submanifold of $E_{n}$ where $n$ is a suitable multiple of $p q$ (Conner and Floyd [4]).

We shall sketch the proofs of (c), (d) in this section and (e) in the next.

(c) By a one point compactification, a given action $\left(Z_{m}, E_{n}\right)$ induces an action $\left(Z_{m}, S_{n}\right)$ in which $\infty \in F\left(Z_{m}\right)$. It is sufficient to show that in any action $\left(Z_{m}, S_{n}\right)$ with $n \leqq 4, F\left(Z_{m}\right)$ can not consist of just one point. This is trivial for $n=0$, easy for $n=1,2$ and straightforward for $n=3$. Consider an action $\left(Z_{m}, S_{4}\right)$. We may assume [21] that $m$ is not the power of a prime. Then $Z_{m}$ contains a subgroup $Z_{p}$ where $p$ is a prime different from 2 . Then $F\left(Z_{p}\right)$ consists of two points or is a homology 2 -sphere over $Z_{p}$. In the second case, enough local properties of $F\left(Z_{p}\right)$ can be established to ensure by a theorem of Wilder [26, Theorem 4.23, p. 223] that it is homeomorphic to $S_{2}$. Thus $F\left(Z_{p}\right)$ is homeomorphic to $S$ where $S=S_{0}$ or $S_{2}$. Now $Z_{m}$ acts on $S$ and the fixed-point set of $Z_{m}$ in $S_{4}$ is identical with the fixedpoint set of $Z_{m}$ in $S_{4}$, hence can not consist of just one point.

(d) We shall sketch the proof of a proposition from which (d) follows readily:

Let $Z_{p q}$ act differentiably on $E_{n}$ and assume that $F\left(Z_{p}\right)$, which is a differentiable submanifold of $E_{n}$, is of dimension $n-2$. Then $F\left(Z_{p q}\right)$ $\neq \varnothing$.

Let $F=F\left(Z_{p}\right)$. The closed differentiable $(n-2)$-manifold $F$ is orientable, connected, and noncompact. Duality relations show that $H_{1}\left(E_{n}-F ; Z\right)=Z$. We consider the linking numbers $l(\xi, \Delta)$ where $\xi$ is an arbitrary integral singular 1-cycle in $E_{n}-F$ and $\Delta$ an integral infinite fundamental $(n-2)$-cycle for $F$. The integer $l(\xi, \Delta)$ depends only on the homology class of $\xi$ in $H_{1}=H_{1}\left(E_{n}-F, Z\right)$. If $t \in H_{1}$ and $g \in Z_{p}$, then $g t=\epsilon t$ when $|\epsilon|=1 ;-1$ can only occur if $p=2$. Hence $l(g \xi, \Delta)=\epsilon l(\xi, \Delta)$ so in any case $l(g \xi, \Delta)=l(\xi, \Delta) \bmod p$, and $(\sigma \xi, \Delta)$ $=0 \bmod p$ where $\sigma=1+g+\cdots+g^{p-1}$. Call a $Z_{p}$-invariant 1 -cycle in $E_{n}-F$ simple if it is expressible as $\sigma \alpha$ where $\partial \alpha=x-g x, x$ a point, $g \neq 1$. If $\eta_{1}, \eta_{2}$ are simple 1 -cycles then $l\left(\eta_{1}, \Delta\right)=l\left(\eta_{2}, \Delta\right) \bmod p$. For say $\eta_{i}=\sigma \alpha_{i}, \partial \alpha_{i}=x_{i}-g x_{i}$. Let $\beta$ be a 1 -chain in $E_{n}-F$ such that $\partial \beta=x_{2}-x_{1}$. Then $\eta_{1}-\eta_{2}=\sigma \zeta$ where $\zeta$ is the cycle $\alpha_{1}-\alpha_{2}+\beta-g \beta$. Hence $l\left(\eta_{1}-\eta_{2}, \Delta\right)=0 \bmod p$. Now $Z_{p}$ acts on $F$ and $F$ is connected. Hence there are simple cycles in $F$. Let $\mu$ be one. If $F \cap F\left(Z_{q}\right)=\varnothing$, then $\mu \subset E_{n}-F$. On the other hand, $H_{1}\left(F\left(Z_{q}\right), Z_{q}\right)=0$ and therefore 
the homology class of $\mu$ (or any integral 1-cycle in $E_{n}-F$ ) is divisible by arbitrarily large multiples of $p$. The same is true of $l(\mu, \Delta)$ which implies that $l(\mu, \Delta)=0$. On the other hand, it follows easily from differentiability that there exist simple cycles $\beta$ in $E_{n}-F$ near $F$ such that $l(\beta, \Delta)=1$. Then since $l(\mu-\beta, \Delta)=0 \bmod p$ we have $l(\beta)$ $=0 \bmod p$ which is impossible. It follows that $F \cap F\left(Z_{q}\right)$, which equals $F\left(Z_{p q}\right)$, is not empty.

Question. Can $F\left(Z_{p q}\right)$ be compact? $\left(F\left(Z_{p}\right)\right.$ and $F\left(Z_{q}\right)$ are not.)

10. Semi-free actions. Let $X_{q}$ be finite dimensional and acyclic with respect to homology over $Z_{q}$. Let $p, q$ be primes. Although there exists no semi-free action $\left(Z_{p}, X_{q}\right)$ when $p=q$, the following example shows that such actions may exist when $p \neq q$.

Let $C$ be a circle with angular coordinate $\theta$ and $\left\{m^{k}\right\}$ the mapping $C \rightarrow C$ of degree $m^{k}$ given by $\theta \rightarrow m^{k} \theta$. Let $\rho_{m}$ be the rotation $\theta \rightarrow \theta$ $+2 \pi / m$. For $t \geqq 0$ let $[t]$ be the largest integer $\leqq t$. On the semiinfinite cylinder $W^{2}=C \times[0, \infty)$ the sets $\left(\left\{m^{[t]}\right\}^{-1} c, t\right), c \in C$, form a decomposition defining an equivalence $\epsilon_{m}$. Let $W_{m}^{2}=W^{2} / \epsilon_{m}$ and consider the subsets $W(t)=(C \times t) / \epsilon_{m}, W[t, s)=(C \times[t, s)) / \epsilon_{m}$ etc. of $W_{m}^{2}$. For $k=0,1, \cdots, W[k, k+1)$ is retractible to $W[k+1]$ by a deformation which maps every $W(t), t \in[k, k+1)$ onto $W(k+1)$ with degree $m$. It follows readily that $W_{m}^{2}$ is acyclic over $Z_{m}$. The rotation $(c, t) \rightarrow\left(\rho_{n} c, t\right)$ of $W^{2}$ induces an action $\left(Z_{n}, W_{m}^{2}\right)$ which is obviously free if $m$ is prime to $n$, as we now suppose. An easy triangulation makes this action simplicial. $W_{m}^{2}$, which is now an infinite 2-complex can be imbedded in $E_{j} \times \cdots \times E_{j}$ ( $n$ factors, $j$ sufficiently large) in such a way [7] that $\left(Z_{n}, W_{m}^{2}\right)$ is induced by a simplicial orthogonal action $\left(Z_{n}, E_{j} \times \cdots \times E_{j}\right)$ where $g(x, y, \cdots, z)=(y, \cdots, z, x), g$ a generator of $Z_{n}$. The group $Z_{n}$ acts freely on the regular neighborhood $U$ of $W_{n}^{2}$ and the action $\left(Z_{n}, U\right)$ is orthogonal ( $\left.\$ 1\right)$. Since $U$ is retractible to $W_{n}^{2}$ by deformation, it is acyclic over $Z_{m}$.

A similar construction by Conner and Floyd [4] gives the semi-free action in (e), §9. We retain the notation of the preceding paragraph. If the segment $x \circ y$ in $C \circ C$ is subdivided into three segments of equal length, there is a unique piecewise linear mapping of $x \circ y$ onto the 1-chain $\left(\left\{m^{k}\right\} x\right) \circ y-x \circ y+x \circ\left\{n^{k}\right\} y$, and the totality of these mappings for all segments $x \circ y$ gives a mapping $\{m, n\}: C \circ C$ $\rightarrow C \circ C$ which is of degree $m-1+n$. Thus $\{m, n\}$ is of degree 0 if $m+n=1$ as we now suppose. On the semi-infinite cylinder $W^{4}$ $=(C \circ C) \times[0, \infty)$, the sets $\left(\left\{m^{[t]}, n^{[t]}\right\}^{-1} c, t\right)$ form a decomposition of $W^{4}$ defining an equivalence $\epsilon_{m n}$. Consider the subsets $W(s, t)$ etc. of $W_{m n}^{4}$. For $\beta=0,1, \cdots, W[k, k+1]$ is retractible to $W[k+1)$ by a deformation which maps $W(t), t \in[k, k+1)$ onto $W(k+1)$. Now 
$W(t)$ and $W(k+1)$ are canonically homeomorphic to $C \circ C$ and the maps $W(t) \rightarrow W(k+1)$ are readily identifiable with $\{m, n\}$ and hence are of degree 0 . It follows that for $k=0,1, \cdots, W[k, k+1)$ is deformable on $W[k, k+1]$ to a point and one can infer from this that $W_{m n}^{4}$ is homotopically trivial, hence contractible. Now let $p, q$ be distinct primes. Then the integers can be chosen congruent to 1 modulo $p$ and $q$ respectively (still with $m+n=1$ ). In this case the transformation $(c, t) \rightarrow\left(\left(\rho_{p} \circ \rho_{q}\right) c, t\right)$ of $W^{4}$ of period $p q$ induces an action $\left(Z_{p q}, W_{m n}^{4}\right)$ which is semi-free and, as in the preceding paragraph, this leads to a semi-free orthogonal action $\left(Z_{p q}, U\right)$ where $U$ is contractible (and $\operatorname{dim} U$ is a multiple of $p q$ ).

QUestion. What is the smallest dimension $U$ can have in such an action?

11. Orbit spaces. Let $(G, X)$ be an effective action. Floyd showed $[5 ; 6]$ that if $X$ is acyclic, one can expect the orbit space $X / G$ to be acyclic. More specifically, if $X$ is a locally compact Hausdorff space and if the compactly supported integral cohomology of $X$ is trivial, the same is true of $X / G$.

In general, however, the computation of the cohomology of $X / G$ appears to be complicated. Let $p$ be a prime In the following proposition we denote by $P(X, t)$ the Poincaré polynomial of $X$ for compactly supported cohomology over $Z_{p}$ and by $Q(a, b)$ the polynomial $t^{a}+t^{a+1}+\cdots+t^{b}$.

Let $G=Z_{p} \times Z_{p}$ and let $X$ be a homology $n$-sphere over $Z_{p}, p$ a prime, and let $G$ act effectively on $X$. Let $Z_{p}^{i}, i=1, \cdots, p+1$, be the non-trivial cyclic subgroups of $G$ and let $n_{i}=\operatorname{dim}_{p} F\left(Z_{p}^{i}\right), i \geqq 1$ and $n_{0}=\operatorname{dim}_{p} F(G)$. The $Z_{p}$-cohomology of the orbit space of the free part of the action is given by

$$
(1-t) P\left(X^{f} / G, t\right)=\sum_{i=1}^{p+1} Q\left(n_{0}+2, n_{i}+1\right)-Q\left(n_{0}+2, n+1\right)
$$

which can be solved explicitly:

$$
P\left(X^{f} / G, t\right)=t^{n_{0+2}} \sum_{i=2}^{p+1} Q\left(0, q_{1}+\cdots+q_{i-1}-1\right) Q\left(0, q_{i}-1\right)
$$

where $q_{i}=n_{i}-n_{0}$. Putting $t=1$ in (2) gives

$$
\sum_{i=1}^{p+1}\left(n_{i}-n_{0}\right)=n-n_{0} .
$$

A formula like (1) for higher powers of $Z_{p}$ has apparently not yet been obtained although a recursion formula has been conjectured 
[23]. Borel [18] however showed that the dimensional relations (3) hold with $n_{i}, i \geqq 1$ defined to be $\operatorname{dim}_{p} F\left(G^{i}\right)$ where $G^{1}, G^{2}, \ldots$ are the subgroups of index $p$.

An Application. Let $\left(Z_{2}, P_{n}\right)$ be an effective action where $P_{n}$ is projective n-space. Then $F\left(Z_{2}\right)$ is empty or else has two components $A_{1}$ and $A_{2}$ where $A_{i}$ is a homology projective $n_{i}$-space over $Z_{2}\left(i . e . H^{*}\left(A_{i}, Z_{2}\right)\right.$ $=H^{*}\left(P_{n_{i}}, Z_{2}\right)$ ), and $n_{1}+n_{2}=n-1$.

Suppose in fact that $F\left(Z_{2}\right) \neq \varnothing$. Then there exists a covering action $\left(Z_{2}, P_{n}\right)$ on the universal covering $\widetilde{P}_{n}=S_{n}$ of $P_{n}$ (cf. the proof in $\S 8$ ) such that $g \tilde{x}$ covers $g x$ when $\tilde{x}$ covers $x$. Let $g$ be the nontrivial element of $Z_{2}$. The transformation $x \rightarrow g x$ permutes with the decktransformation $t: P_{n} \rightarrow P_{n}$ and hence $g, t$ define an action $\left(Z_{2} \times Z_{2}, P_{n}\right)$ whose fixed point sets we denote by $\widetilde{F}$. Of course $\widetilde{F}(t)=\varnothing$ so $\tilde{F}\left(Z_{2} \times Z_{2}\right)=\varnothing$. Then $\widetilde{F}\left(Z_{2} \times Z_{2}\right), \tilde{F}(t), \tilde{F}(g), \tilde{F}(t g)$ are homology spheres over $Z_{2}$ of dimensions $-1,-1, n_{1}, n_{2}$ respectively and by the preceding proposition we have $n_{1}+n_{2}=n-1$. Now if $\tilde{x}$ in $P_{n}$ is fixed under $g$, then $\phi^{-1} x=(\tilde{x}, t \tilde{x})$ (where $\phi$ is the projection $\widetilde{P}_{n} \rightarrow P_{n}$ ) is invariant under $g$ so that either $g \tilde{x}=\tilde{x}$ or $g t \tilde{x}=\tilde{x}$. It follows that $F(g)=\phi(\widetilde{F}(g) \cup \widetilde{F}(g t))$. Now $\widetilde{F}(g) \cap \widetilde{F}(g t) \subset \widetilde{F}(g g t)=\widetilde{F}(t)=\phi$ and in fact $t \tilde{F}(g) \cap \widetilde{F}(g t)=\varnothing$ since $t \widetilde{F}(g)=\widetilde{F}(g)$. Hence $F(g)$ in the disjoint union of $\phi \widetilde{F}(g)$ and $\phi \widetilde{F}(g t)$, which may be regarded as orbit spaces of free actions of $Z_{2}$ on the homology spheres $\widetilde{F}(g), \widetilde{F}(g t)$ of dimensions $n_{1}$ and $n_{2}$, hence have the stated cohomology.

QUESTION. Is this proposition true if it is assumed only that $P_{n}$ is a homology projective space over $Z_{2}$ ?

\section{REFERENCES}

1. R. H. Bing, A homeomorphism between the 3-sphere and the sum of two solid horned spheres, Ann. of Math. vol. 56 (1952) pp. 354-362.

2. A. Borel, Nouvelle démonstration d'un théorème de P. A. Smith, Comment. Math. Helv. vol. 29 (1955) pp. 27-39.

3. H. Cartan and S. Eilenberg, Homological algebra, Princeton, Princeton University Press, 1956.

4. P. E. Conner and E. E. Floyd, On the construction of periodic maps without fixed points, Proc. Amer. Math. Soc. vol. 10 (1959) pp. 354-360.

5. E. E. Floyd, Orbit spaces of finite transformation groups. I, Duke Math. J. vol. 20 (1953) pp. 563-567.

6. - Orbit spaces of finite transformation groups. II, Duke Math. J. vol. 22 (1955) pp. 33-38.

7. - Fixed point sets of compact abelian Lie groups of transformations, Ann. of Math. vol. 66 (1957) pp. 30-35.

8. E. E. Floyd and R. W. Richardson, An action of a finite group on an n-cell without stationary points, Bull. Amer. Math. Soc. vol. 65 (1959) pp. 77-79.

9. J. J. Greever, Fixed points of finite transformation groups, Dissertation, University of Virginia. 
10. M. W. Hirsch and Stephen Smale, On involutions on a 3-sphere, Notices, Amer. Math. Soc. vol. 6 (1959) pp. 148-149.

11. S. Lefschetz, Algebraic topology, Amer. Math. Soc. Colloquium Publications, vol. 27, 1942.

12. B. von Kérékjártó, Über die endlichen topologischen Gruppen der Kugelfäche, Nederl. Akad. Wetensch. Proc. Ser. A vol. 22 (1919) pp. 568-569.

13. J. Milnor, Groups which act on $S_{n}$ without fixed points, Amer. J. Math. vol. 79 (1957) pp. 623-630.

14. D. Montgomery and H. Samelson, $A$ theorem on fixed points of involutions in $S^{3}$, Canad. J. Math. vol. 7 (1955) pp. 208-220.

15. C. D. Papakyriakopolous, On Dehns' lemma and the asphericity of knots, Ann. of Math. vol. 66 (1956) pp. 1-26.

16. G. de Rham, Sur les nouveaux invariants topologiques de M. Reidemeister, Recueil Math. (Moscow) new series, vol. 1 (1936) pp. 737-742.

17. H. Seifert and W. Threlfall, Topologische Untersuchung der Diskontinuitätsbereiche endlicher Bewegungsgruppen des dreidimensionalen sphärischen Raumes. I, Math. Ann. vol. 104 (1930) pp. 1-70.

18. Seminar on transformation groups, Institute for Advanced Study, 1958-195.

19. P. A. Smith, Transformations of finite period, Ann. of Math. vol. 39 (1937) pp. $137-164$.

20. —, Transformations of finite period. II, Ann. of Math. vol. 40 (1939) pp. 690-711.

21. - Fixed point theorems for periodic transformations, Amer. J. Math. vol. 63 (1941) pp. 1-8.

22. —, Permutable periodic transformations, Proc. Nat. Acad. Sci. U.S.A. vol. 30 (1944) pp. 105-108.

23. - Orbit spaces of abelian p-groups, Proc. Nat. Acad. Sci. U.S.A. vol. 45 (1959) pp. 1772-1775.

24. R. G. Swan, Groups with periodic cohomology, Bull. Amer. Math. Soc. vol. 65 (1959) pp. 368-370.

25. J. H. C. Whitehead, An involution of spheres, Ann. of Math. vol. 66 (1957) pp. 27-29.

26. R. L. Wilder, Topology of manifolds, Amer. Math. Soc. Colloquium Publications, vol. 32, 1949.

27. H. Zassenhaus, Theory of groups, New York, Chelsea Publishing Co., 1949.

28. —- Über endliche Fastkörper, Abh. Math. Sem. Univ. Hamburg vol. 11 (1936) pp. 187-220.

\section{Columbia University}

